\title{
ANDA Dissolution Method Development and Validation
}

Intraduetion

Although the title of this manuscript refers to " $A N D A$

Dissolution Method Development and Validation," the primary

focus of the manuscript will be the validation aspect. This, of course, is because an ANDA filing is most often for a generic product for which a dissolution method bas already been developed and is usually in the USP and/or available from the regulatory authorities. Therefore, I will primarily address the validation process which a company filing an ANDA should perform in order to obtain product approval. There is, however, a situation where a multisource product manufacturer will be required to develop new dissolution methodology. I will comment on this later in the discussion.

Since validation of a dissolution procedure is comparable to the validation of any of a company's analytical methods, one might simply refer back to the various analytical validation procedures in their company's Standard Operating Procedures (S.O.Ps) which can be applied to dissolution, and combine them into a new S.O.P. In order to present a multifaceted view, I contacted a number of companies, both innovator and generic, to determine the process which they employed. What you are reading is an amalgamation of my experiences and their various procedures.
Although I previously indicated that validating a dissolution procedure is little more than validating any of a company's analytical methods, there is one major difference which makes it unique. Unlike all other analytical procedures, there is no absolute standard available to validate a dissolution method. Some workers like to consider USP calibrators as the absolute standard but, in fact, they only represent an adjunct part of an overall process which bopefully validates the procedure as well as the more classical analytical method validation processes are able to do.

The author is indebted to colleagues at Ciba-Geigy Corporation, G.D. Searle and Company, Sidmak Laboratories, and Zenith Laboratories, who generously supplied me with their respective companies' dissolution validation procedures.

I view validation of any dissolution procedure as a two-step process. The first step consists of separately validating only the dissolution apparatus whereas the second step requires validation of the specific dissolution procedure itself. Without both of these being performed, I do not consider the method to be adequately validated. I will therefore consider these two steps separately.
Lewis ل. Leeson, Ph.D.

L7L Associates, Inc., Montville, N7

Presented at the AAPS Dissolution Short Course in Seattle, Washington on October 27, 1996

\section{The Apparatus}

Most, if not all, of the official dissolution methods as well as others described in the literature, even if they yield different results, are constructed in essentially the same way.

The component parts are:

1. The Container

2. The Stirrer

3. The Dissolution Medium

4. The Operating Temperature Control

5. The Detection/Analysis System

Because, as I indicated previously, there is no absolute standard for dissolution methods, one must in a sense validate the apparatus indirectly. My approach to doing this is to prepare an S.O.P. to assure that the key factors of the apparatus are carefully defined in order to assure reproducible performance. The first thing to be done is to go through the component parts of each apparatus to be certain that everything is in accord with good science and USP requirements. It should be emphasized that a major component of the S.O.P. is good record keeping. The S.O.P. serves 2 purposes: (1) To assure that everyone in the laboratories is validating the equipment in the same way, and (2) To assure the regulatory authorities of the quality of your dissolution validation process.

When validating the apparatus, it is extremely important to operate the equipment as defined in the USP. Many of the following considerations have to be addressed only once, while others may require daily performance. Experience and a sound scientific approach will help determine the proper time schedule. The following is a brief summary of the items to consider during the preparation of an S.O.P. for Apparatus Validation.

\section{The Container}

The container's dimensions must be in accord with the specifications in the USP(1). This applies to the kettles used with Apparatus 1 and 2, the cylinder and vessel used for Apparatus 3 , and the cells used with Apparatus 4. One cannot assume that the dimensions are correct because the vessel manufacturers

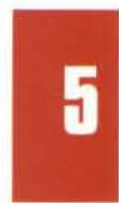




\section{ANDA Dissolution Method ... cont.}

say so. Rather they should be measured at the time of receipt, and the results documented by the user. In addition to the source of the kettles, it is important to note whether they are plastic or glass and whether those made of glass are molded or were formed from tubing. Although this latter information may not be critical, it is valuable to have in regard to possible influence on method performance. Finally, one should also check the seating of each vessel in the apparatus to be certain that it is level and that the cover fits properly to prevent evaporation of dissolution medium.

\section{The Stirrer}

Like the container, the stirrers on Apparatus 1 and 2 must meet the physical specifications described in the USP(1). In addition, the screen on the rotating basket must be welded and of the proper mesh $(40 \times 40$ or $20 \times 20)$. When placed in position there must be no significant wobble in the shaft. In the case of Apparatuses 1 and 2, the position for each spindle should be well defined so that the basket or paddle is seated at a distance from the vessel's bottom in accord with USP specifications. The validity of the speed settings should be verified with a tachometer. In the case of Apparatus 3 the accuracy of the reciprocating rate settings and distance should be established. With Apparatus 4, the pump speed settings should be verified as well as demonstrating that the pump has the proper flow profile (sinusoidal pulsation). It is often of value to measure vibration at this point both with and without the pumps and motors functioning.

\section{The Dissolution Medium}

Validation of a buffered dissolution medium is essentially based upon preparing the correct buffer, and employing a properly standardized validated $\mathrm{pH}$ meter. This is a routinely defined procedure in all laboratories. If, however, the $\mathrm{pH}$ of the dissolution medium is temperature dependent, both the determination of the medium $\mathrm{pH}$ and standardization of the $\mathrm{pH}$ meter must be performed at the dissolution method's operating temperature. In addition, a method for adding the proper amount of dissolution medium to a each vessel must be carefully defined (Is it added by weight or volume?) and be part of a written procedure for preparation and use of the dissolution medium. This procedure should also include your specific method for deaeration of the dissolution medium $(2,3,4,5)$. One suggestion in this regard is to consider a method which was employed by one group at CIBA-Geigy. They prepared large volumes of all commonly utilized dissolution media in advance and maintained them at $37^{\circ} \mathrm{C}$. It was determined that these solutions could be used as is, since they were self-deaerating.

\section{The Operating Temperature}

Temperature, like $\mathrm{pH}$ determination, is such a common measurement in laboratories that an S.O.P. for validating it is likely to be available at every site. One may employ something as simple as an ASTM standard thermometer to validate the Apparatus' thermometers or as sophisticated as utilizing a validated computerized feedback control system. Both can be equally satisfactory. One point to be kept in mind would be the timing to help ensure that the contents of each vessel has achieved the desired temperature. Because of the pressure to perform the large amount of dissolution being required today, timing is often used as a measure to establish that the medium is at the proper initial temperature. For example, different time factors will obviously be required for a dissolution medium prepared, stored at room temperature and deaerated prior to use, and one which is stored under heated conditions and may not require deaeration. In addition, one configuration of USP Apparatus 4 has a heating coil as part of the flow system. The purpose of the coil is to raise the temperature of the dissolution medium to the desired value. If a laboratory employs this configuration, it should be demonstrated that the timing will be such as to allow the proper temperature to be attained.

\section{The Detection/Analysis System}

Of all the components of a dissolution apparatus, I believe the one with which the analysts are most comfortable is the Detection/Analytical System, which, unlike many of the components is utilized in a myriad of other analyses. Although a number of Detection/Analytical Systems have been employed as part of dissolution equipment, the most common have been direct UV-VIS Spectrophotometry and High Performance Liquid Chromatography (HPLC) in conjunction with photometric detection. Validation of the functioning of this equipment is readily defined in the operations manuals supplied by the manufacturer. Such things as wavelength accuracy, temperatures, flow rates, noise, etc. are discussed and methods of optimizing performance are presented. 
Validation of such equipment is associated with the S.O.P. for its utilization. As part of this, a proper operation/repair record must be maintained.

The final step in validating the dissolution apparatus is the utilization of the USP calibrators. As indicated previously, there are no absolute standards for dissolution. However, the USP Calibrators represent the closest thing that we have to them. These formulations are specially prepared for use in calibrating USP dissolution equipment. USP Apparatus 1 and 2 utilize both prednisone (disintegrating tablet) and salicylic acid (non-disintegrating) tablets. For USP Apparatus 3, Chlorpheniramine Maleate ExtendedRelease Tablets and Theophylline Extended-Release Beads are employed. The validation conditions and specifications are supplied with the samples whenpurchased from the USP. I am aware that there is controversy over whether the USP prednisone tablet or the FDA Prednisone NCDA\#2 is the better one to utilize. I don't have a bias for either, but only propose that the one which the USP ultimately selects should be employed. It must be remembered, however, that there are possible pitfalls associated with proper dissolution calibration. These have been discussed in various publications $(2,6)$.

\section{The Method}

In my opinion, validation of the specific dissolution method itself tends to be easier than validating the apparatus. This is because much of validating the method relates to the detection system, a procedure which is probably already defined by an existing S.O.P. As in the case of apparatus validation, there should be a written document clearly describing the exact dissolution method to assure that each analyst performs it in the identical manner. This document will describe apparatus, dissolution medium, mixing speed, sampling times, and the specific analytical method utilized. Since most of these aspects are part of the Apparatus Validation process, the most important feature for the dissolution procedure validation is that of the analytical method itself. The typical parameters used to accomplish this are listed in the USP(7). These are:
1. Accuracy
2. Precision (within and between laboratories and analysts)
3. Specificity
4. Limit of Detection/Limit of Quantitation

\section{Linearity \\ 6. Range \\ 7. Ruggedness}

It should be kept in mind that if one is utilizing an already validated method, for example, one developed for analysis of pure active ingredient, many of the above parameters have already been validated. It is therefore only necessary to perform those that are specific for the dissolution method analyte determination such as specificity or any analysis that includes procedures which are different from the existing method.

The parameters indicated above are classic and utilized to validate any analytical procedure. I therefore suspect that the reader is familiar with their evaluation. However, they will be discussed briefly for the sake of completeness.

Accuracy is defined as the closeness of the result to the correct value. It is a measure of the exactness 


\section{ANDA Dissolution Method ... cont.}

of the analytical method, and is expressed as a percent of the actual known amount added in test mixtures.

Precision is the degree of agreement between individual test results when the test is applied to multiple sub-samplings of the same sample. It may be considered in light of reproducibility of results from a single analyst, between analysts in the same laboratory or between analysts in different laboratories. It is expressed in terms of standard deviation of the analyte mean results calculated at each known test concentration. One multiple design approach utilized by a major American company to obtain such information is as follows:

Design A - One analyst on two days with two runs per day.

Design B - Two analysts and four days with one run per day.

Design C - Four analysts and four days with two runs/day/analyst.

A full statistical evaluation of the results from such a design will produce much more information about the performance of the method than just precision. The designs indicated above also supply some information as to ruggedness of the method.

Specificity represents the ability of the analytical method to measure accurately and specifically only the analyte in the presence of components which might be expected to be found in the sample being analyzed. It is the degree of interference in analysis of a complex mixture, and represents a bias which could be introduced by various impurities present in the mixture such as formulation excipients, impurities, degradation products, or other related compounds. The bias is expressed by the difference in actual value from the determined value. The major difference between specificity and precision is that specificity variability is always in one direction.

Limit of Detection is the lowest concentration of an analyte that can be detected, but not necessarily quantitated, under a given set of conditions. It is merely used to indicate that the amount detected is above or below a certain level and is usually the point at which the signal to noise ratio is from $2: 1$ to $3: 1$. It differs from the limit of quantitation which is the lowest concentration of analyte that can be detected with acceptable precision and accuracy under the stated experimental conditions. It is determined to be 10 times the standard deviation of the assay of a series of blank samples.
Linearity is the degree to which the response, or some well defined mathematical transform of the response, measured by the method, is directly proportional to the concentration of analyte in a sample. It is expressed in variance of the regression line slope calculated from the data. In the case of a dosage form, the linearity is often determined over the range of $10 \%$ to $120 \%$ of the amount expected to be released during the dissolution run.

Range is the set of concentrations bounded on the lower side by the limit of quantitation and on the upper side by highest amount of analyte demonstrating linearity. The range is expressed in the same units as the test results.

Ruggedness is the degree of reproducibility of the results obtained by the analysis of the same samples under a variety of normal conditions such as: a) different laboratories, b) different analysts, c) different days, d) different instruments, e) different instrument temperatures, f) different UV wavelengths, etc. Even if this parameter has already been evaluated for pure active ingredient, it should be determined for the overall dissolution method by stressing key dissolution parameters. Some possible ones are: a) apparatus, b) operator c) deaeration, d) vibration, etc. It is evaluated by comparing the accuracy and precision of the results obtained under both "normal" and "stressed" conditions. In the case of the overall dissolution procedure, this measure of ruggedness is usually called robustness.

In addition to what we have discussed, there are items which do not quite fit or fit only tangentially into the above validation categories. I refer to such things as demonstrating the adequacy of filters or tubing which might be utilized as part of an automated flow system. In addition, although solution stability of the active ingredient may have been demonstrated as a single entity, one must also demonstrate its solution stability in the presence of the formulation's excipients. Although seemingly apparent, I have encountered cases where one or more of these factors have been overlooked.

Although what I have discussed above covers the key factors in validating a dissolution procedure, there are three more points which I would like to address briefly. The first relates to automation of a dissolution method which was originally developed and validated as a unit operation. When this occurs in a company, the newly developed automated method must be treated as a non-validated procedure, and therefore a "revalidation" must take place. This new validation 
is really only applicable to those parts of the procedure that are different from the original. For example, there is no reason to revalidate the basic dissolution equipment itself. However, for UV analyses which will utilize a flow cell, such things as tubing and filter characteristics, flow rate, position of tubing in the dissolution vessels, and temperature in the cell holder must be validated. Corresponding special features, such as automatic sampling procedures, calculations, etc., relating to an automated HPLC procedure must also be addressed. In addition, accuracy and precision must be evaluated to see if the automated and non-automated systems are performing in a similar fashion.

The second point on which I would like to comment is that of revalidation. A particular dissolution method is normally validated only once! The results obtained in the original study are documented in a validation report. There is no need to repeat the validation periodically or at certain occasions, for instance in case of transfer of the test method to another laboratory, as long as the test method is performed strictly according to the testing instructions and the manufacturing process of a product or the product's formulation is not changed. Revalidation is defined as repetition of the validation process, or a specific portion of it, when the aforementioned conditions do not apply. As in my preceding comments with respect to automation, the parts to be revalidated are based on a case by case analysis.

My final point relates to a comment which was made earlier in this report. At that time it was stated that when a company is seeking an ANDA, it starts out with a defined dissolution method either from the FDA or USP. However, this is not always the case, since there may be occasions when the applicant demonstrates bioequivalence of its ANDA product to that of the innovator company, but the product is unable to meet the existing dissolution specification. This is often encountered with extended release products. The ANDA applicant has two options. The first is for the applicant to define the specifications for its product based upon the product's performance with the existing procedure. The company may then request the FDA and USP to change the specifications based upon their data. I personally have doubts about the success of this strategy, since it would require the specifications to be made less stringent. The second option is for the applicant to employ the same dissolution procedure but request that they be permitted to utilize their own dissolution specifications which are different from

the existing product(s).

Alternatively, they could develop a different procedure and specifications, and file them with both the FDA and USP. As a result, for some drug products there could exist multiple product specific specifications, and/or multiple product specific dissolution methods and specifications which are acceptable to both the FDA and USP. This latter situation presently exists for a number of USP products. One example is Theophylline ER Capsules for which there are presently seven different dissolution procedures and/or specifications.

In conclusion, although this document is supposed to be on the subject of the validation of ANDA products, in the author's opinion they should not be any different from what is utilized for NDA products. It should be recognized, that this is only one possible approach, and there are certainly others which are equally good, and possibly better. Those which I have reviewed in preparation for this presentation, although having differences, are essentially similar to this and to each other.

One question which has been asked on a number of occasions is how does one validate an in vitro/in vivo correlation. My reply has always started out with the statement that "you must first begin with a validated dissolution method." I still believe that to be true and hope that the information in this document will help to satisfy that need. It will, however,

See ANDA continued on page 18 
ANDA continued from page 9

require another paper to address the rest of the answer to that question.

\section{References}

1. USP 23/NF 18, <711>, <724>, 1985 .

2. Gray, V.A. and Hubert, B.B., Pharm. Forum, 20:6(1994)8571.

3. Robrs, B.R. and Seltzer, D.F., Diss. Tech., 2, Issue 2, page 1. Spring 1995.

4. Moore, T.W., Ibid, 3, Issue 2, page 3, May 1996.

5. Qureshi, S.A. and McGilveray, I.F., Pharm. Forum, 20:6(1994)8565.

6. Martin, G.P. et al., Diss. Tech., 3, Issue 1, February 1996.

7. USP $23 / \mathrm{NF} 18,<1225\rangle, 1995$. 